Article

\title{
Neuro-Fuzzy Dynamic Position Prediction for Autonomous Work-Class ROV Docking
}

\author{
Petar Trslić *, Edin Omerdic $(\mathbb{D}$, Gerard Dooly and Daniel Toal \\ Centre for Robotics \& Intelligent Systems, University of Limerick, V94 T9PX Limerick, Ireland; \\ edin.omerdic@ul.ie (E.O.); gerard.dooly@ul.ie (G.D.); daniel.toal@ul.ie (D.T.) \\ * Correspondence: petar.trslic@ul.ie; Tel.: +353-61-213-102
}

Received: 21 December 2019; Accepted: 23 January 2020; Published: 27 January 2020

check for updates

\begin{abstract}
This paper presents a docking station heave motion prediction method for dynamic remotely operated vehicle (ROV) docking, based on the Adaptive Neuro-Fuzzy Inference System (ANFIS). Due to the limited power onboard the subsea vehicle, high hydrodynamic drag forces, and inertia, work-class ROVs are often unable to match the heave motion of a docking station suspended from a surface vessel. Therefore, the docking relies entirely on the experience of the ROV pilot to estimate heave motion, and on human-in-the-loop ROV control. However, such an approach is not available for autonomous docking. To address this problem, an ANFIS-based method for prediction of a docking station heave motion is proposed and presented. The performance of the network was evaluated on real-world reference trajectories recorded during offshore trials in the North Atlantic Ocean during January 2019. The hardware used during the trials included a work-class ROV with a cage type TMS, deployed using an A-frame launch and recovery system.
\end{abstract}

Keywords: ANFIS; ROV docking; Position prediction

\section{Introduction}

In recent years, operations undertaken by unmanned underwater vehicles (UUVs) in the offshore energy sector are changing rapidly. This is driven by both offshore oil \& gas $(O \& G)$ and the offshore wind sector where production platforms are pushed further off the coast, into areas of higher energy potential. However, considering significant expenditures related to the cost of the surface support vessel and crew, and with the production platforms in remote locations, the cost related to inspection, maintenance, and repair (IMR) tasks inevitably rise. Rising costs have resulted in the development and use of permanently deployed resident vehicle systems. Although the concept of permanently deployed vehicles exists in the literature for many years [1], only recently have we seen the introduction of commercial resident vehicles [2], with Oceaneering and IKM being industry leaders. Oceaneering developed E-ROV [3], a battery-powered, self-contained, work-class remotely operated vehicle (ROV), whereas IKM developed a fully electric R-ROV based on electric work-class ROV Merlin [4]. In general, such systems include a permanently deployed docking station which serves as a charging point, download/upload data link, and as mechanical protection for the resident vehicle [5].

However, within the $O \& G$, and especially the offshore wind production field, multiple assets can be spread across more than $100 \mathrm{~km}^{2}$, which need to be continuously inspected for condition monitoring purposes. This has been partially addressed through the development of resident autonomous underwater vehicles (AUV) [6,7]. However, due to the limited intervention capabilities of resident AUV systems, many energy-intensive applications still require ROVs [8]. These restrictions are recognized, and use of collaborative platforms consisting of an autonomous surface vehicle (ASV) and ROV are seen as a potential solution $[9,10]$. Although commercially available solutions based on observation class ROVs exist [11], significant commercial uptake of the technology is not yet recorded. 
Resident ROVs operating from shore fundamentally demand a high bandwidth, low latency communications link that is often unavailable, thus high levels of automation are needed. This is especially important for time-critical tasks since manual operation from the shore due to the mentioned communication problems is not viable. One of the essential time-critical tasks in resident vehicle operation is the docking of the vehicle at the end of the mission. Autonomous docking of UUVs is a well-researched area, with main focus on docking to a static docking station, both for ROV docking to a tether management system (TMS) [12,13] and AUV docking to a docking station [14,15]. However, a TMS suspended from a surface platform such as a surface vessel, presents a highly dynamic system, with wave height and period dictating the viability of launch and recovery operations [16].

Although docking of UUVs to a moving docking station is reported, the research is mainly focused on an AUV docking and on compensation of disturbances in the horizontal plane (e.g., cross-current), while assuming minimal docking station heave oscillations. Recovering of an AUV by another AUV in shallow water is presented in [17]. The system consists of a "mother" AUV with a funnel shaped docking station attached to its body, designed to accommodate launch and recovery of the "daughter" AUV. A docking to an active docking station is presented in [18]. The paper presents a cooperative guidance system for the AUV docking, whereas the system consists of a funnel shaped receptacle with an active heading adjustment. A USV-based automated launch and recovery system (LARS) for AUVs is presented in [19]. The recovery system is based on the deployment of a thin line with a depressor wing from the surface vessel, whereas the AUV is equipped with a pincer-type mechanism for latching. Another fixed-wing depressor-based solution is presented in [20], where a funnel shaped DS is attached to the depressor and towed by a surface vehicle at a constant speed, whereas the AUV intercepts the docking station and performs the docking. The ROV launch and recovery from an ASV has been previously reported in [21]. The system included Video Ray micro-ROV launch and recovery directly, without the docking station.

One of the major limitations of the autonomous ROV docking to a suspended TMS is the TMS heave motion, which can exceed amplitudes of $3 \mathrm{~m}$. Those limits were recognized and reported during previous trials, which included, a first autonomous docking of a work-class ROV to a suspended TMS [22]. Findings acquired during those trials investigating TMS behavior in a real-world environment and associated docking limitations, have served as a motivation for this paper. Although a certain amount of misalignment between the ROV and the TMS is allowed during docking, work-class ROVs are generally underpowered, and not agile enough to match the TMS heave motion. During the manual docking process this is compensated by the pilot's TMS heave motion analysis, prediction of TMS heave motion, and experience. However, considering the offshore marine renewable energy (MRE) sector with devices placed in areas of strong wind, current, and tides, while acknowledging the previously mentioned communication related problems, manual docking operation from shore is not viable. Also the autonomous docking of a ROV to a garage supported beneath a floating platform is expected to be very challenging. Therefore, to allow for autonomous work-class ROV docking in higher sea states a TMS heave motion prediction method has been developed. To the author's knowledge, this is the first time TMS heave position prediction has been proposed.

This research paper presents development and evaluation of the method for suspended TMS heave motion prediction, based on an adaptive neuro-fuzzy inference system (ANFIS). In addition, the paper discusses the mapping of surface vehicle motions to the suspended docking station coordinate frame. The prediction of TMS heave motion has the potential benefits of allowing autonomous docking in higher sea states, extending the ROV operational weather windows, and reducing the misalignment between the ROV and TMS during the docking process, thus reducing the impact on the ROV system and extending the ROV operational life. Furthermore, the method has a dual benefit of being applicable to autonomous docking or as an aiding tool for the pilot. The ANFIS performance is evaluated on a real-world dataset recorded using a work-class ROV with corresponding cage type TMS, deployed during offshore trials in the North Atlantic Ocean. 


\section{The Hardware}

The significant restricting factor in ROV operations and associated operational weather windows relates to the launch and recovery of the vehicle. This includes both launch and recovery of the ROV from the vessel to the sea, and launch and recovery of the ROV from the TMS while underwater. In this paper problems associated with the latter are discussed. The main systems involved in the ROV deployment trials are a surface vessel, a launch and recovery system (LARS), a tether management system (TMS) and the ROV itself. The overview of the system used during the trials is shown in Figure 1, whereas the basic technical specification is given in Table 1. The ROV used during the trials is a work class Comanche ROV developed by Sub-Atlantic and Forum Energy Technologies, which is one of the standard ROVs used in the offshore sector. The ROV is equipped with two Schilling Orion hydraulic arms and is capable of operating in depth up to $2000 \mathrm{~m}$. The vehicle weights approximately 1.6 tons and can achieve a maximum speed of 2.5 knots.

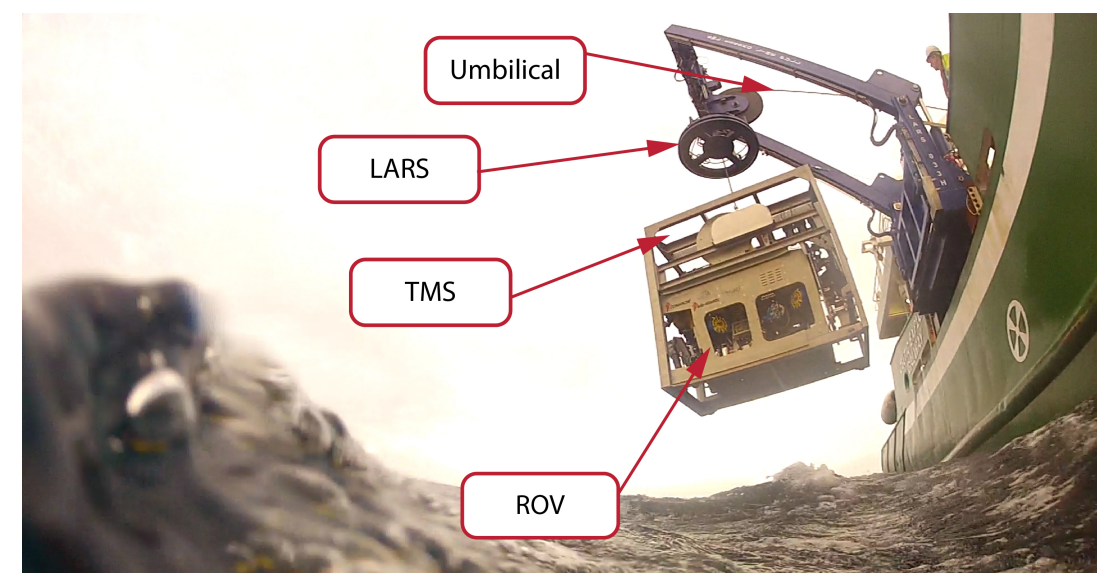

Figure 1. The system deployment during offshore trials in the North Atlantic Ocean in January 2019. The system consists of Launch and Recovery System (LARS), Tether management System (TMS), and work-class ROV Comanche.

Table 1. Technical specification of the system.

\begin{tabular}{llcc}
\hline & Description & Dimensions $\mathbf{L} \times \mathbf{W} \times \mathbf{H}(\mathbf{m})$ & Weight $(\mathbf{t})$ \\
\hline Control Cabin & Reinforced container used as ROV control centre & $6 \times 2.4 \times 2.4$ & \\
LARS & A - frame type, 2200 m steel enforced umbilical, $\phi 25.4 \mathrm{~mm}$ & $5.5 \times 2.8 \times 3.2$ & 2.5 \\
TMS & Cage-type, 400 m soft tether, $\phi 21 \mathrm{~mm}$ & $2.9 \times 1.8 \times 2.5$ & 12 \\
ROV & Middle size ROV capable of inspection, maintenance and repair tasks & $2.1 \times 1.3 \times 1.25$ & 2.2 \\
Ship & Research Vessel & Length - 66 m & Displacement - 2425 t \\
\hline
\end{tabular}

There are various definitions of a tether management system, and although the TMS is in essence only a subsea tether-handling mechanism, by common convention and according to The ROV Manual [23] it is "typically described as the entire subsea mechanism from the end of the umbilical (umbilical termination to the clump/depressor weight, cage, or top hat) to the beginning of the soft tether". There are two main types of tether management systems, the top hat TMS and the cage type TMS. Although most discussed problems related to TMS heave compensation could relate to both types of TMS, in the remainder of this paper when using term TMS, a cage-type TMS is assumed.

There are three main functions of the tether management system: (1) to manage, usually neutrally buoyant soft tether, which connects the TMS and the ROV, and provide power and communications to the ROV; (2) to protect the vehicle against damage during ROV deployment and recovery phase; and (3) to act as a clump weight to absorb the cross-section drag that would be otherwise introduced to a tether connecting ship and the ROV. Therefore, the ROV is completely relieved of the tether drag from the surface to the working depth, which is important due to the limited power on-board the ROV available for vehicle drag compensation caused by sea currents. To act as a clump weight, the TMS has to be negatively buoyant; thus, as a consequence, the ship's motion is directly translated to the TMS 
through a steel reinforced umbilical connecting the TMS with the ship. The TMS used during the trials is a cage type, side entry TMS and weights approximately 2.2 tons.

The LARS system is used as the overboarding equipment, and its primary role is to move ROV from the deck and deploy it safely. The most typically used LARS is the A-frame type, such as the one used during the trials. The system weights around 12 tons and contains $2.2 \mathrm{~km}$ of umbilical. On one side the LARS is connected to the control cabin and ship's power supply, and on the other side to the TMS through the umbilical. The umbilical used for TMS deployment is steel reinforced for lifting to/from the water, and it provides the power and communication link between the ROV and the control cabin. Vessels that are specially designed for ROV operations may include the moonpool or Cage \& Rail LARS system [9] for deployment. However, such equipment is more complicated, thus more expensive, and generally is used as a permanent feature on the vessel. The control cabin is considered the ROV control center with multiple PCs dedicated to ROV control, sonar imaging, image acquisition, processing, etc. Therefore, the data acquired by ROV sensors is sent to the control cabin on the surface where all computation related to the ROV operations is conducted. During the trials the complete system was deployed on the $66 \mathrm{~m}$ long research vessel RV Celtic Explorer.

\subsection{The TMS Motion Analysis}

The TMS with the ROV is usually deployed from a surface vessel or floating platform which is exposed to various disturbances such as waves, currents, wind, tides, and others. As the surface vessel for TMS deployment is the main source of the TMS motion, it is necessary to understand all the disturbances introduced to the surface vessel and how they map to the TMS. As the disturbances act on all six degrees of freedom (DOF) of the surface vessel, and considering the TMS is connected with the vessel through the non-elastic umbilical, those motions couple to the TMS directly. Therefore, the primary goal during the TMS deployment is to minimize the impact of those disturbances on a surface vessel.

Figure 2a shows a vessel's six degrees of freedom. Work-class ROV operations generally imply use of a deployment vessel with dynamic positioning (DP) capabilities. A DP vessel is capable of holding position and heading, thus sea current and wind-related disturbances are bounded by vessel's surge, sway, and yaw control. However, the sea-wave height and period have a direct impact on vessel's roll, pitch, and heave, which cannot be directly compensated for; thus, these remaining three DOF translate to TMS principally as a heave motion. Figure $2 b$ shows the ship motion translated to the TMS.

a)

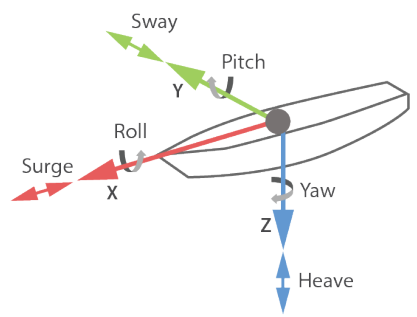

b)
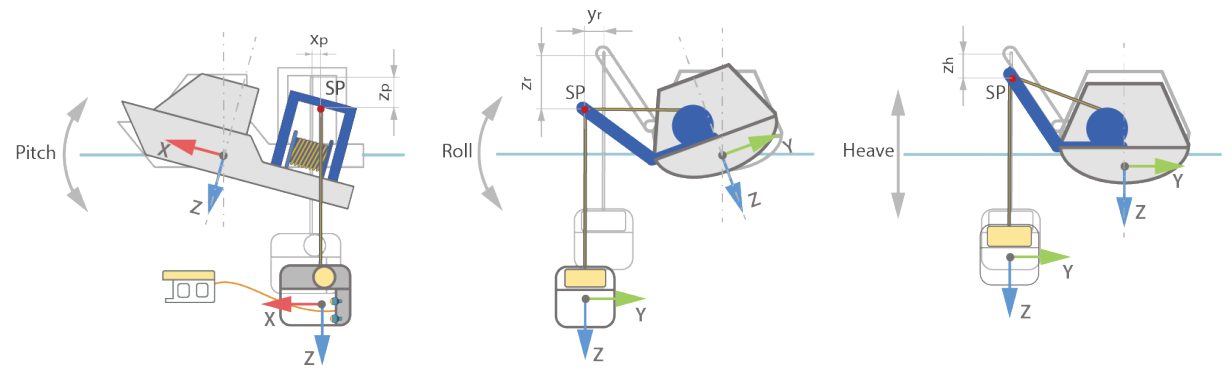

Figure 2. Ship motion mapped to the TMS heave motion. (a) Vessel's six degrees of freedom; (b) Deployment vessel pitch and roll mapped in TMS coordinate frame. 
Given the ship heave, roll, and pitch, a total TMS heave displacement $z_{\mathrm{TMS}}$ is calculated as

$$
z_{\mathrm{TMS}}=z_{\mathrm{h}}+z_{\mathrm{r}}+z_{\mathrm{p}}
$$

where $z_{\mathrm{h}}$ is heave of the TMS directly proportional to the heave of the ship, $z_{\mathrm{r}}$ is heave of TMS due to the ship roll motion, and $z_{\mathrm{p}}$ is the heave of the TMS due to the ship pitch. As shown in the figure, to reduce the TMS heave, the suspension point SP should ideally be placed close to the ship pitch and roll axis. The vessels designed specifically for ROV operations exploit this with integrated, moonpool LARS or ship door LARS [16,23] systems. However, the most typical LARS is the A-frame type, as shown in Figure 1.

The docking station is relatively stable on the roll and pitch axis since the TMS center of gravity is below the point where TMS is attached to the umbilical, thus positive longitudinal and lateral stability is achieved. Sea current generally rotates the TMS around the yaw axis, until TMS reaches the orientation that creates the least amount of drag. However, TMS yaw is easily controllable with two or more thrusters attached to the TMS. There are two sources of TMS surge and sway: (1) surface vessel surge and sway which depends on DP capability, and (2) the TMS surge and sway caused by displacement of suspension point $y_{r}$ and $x_{p}$ due to vessel's roll and pitch, which, for relatively small angles can be neglected. In addition, the TMS inertia, length of the deployed umbilical, and water act together as a damper, thus they reduce surge and sway oscillations.

In summary, the suspended TMS heave displacement $Z_{T M S}$ depends on the surface vessel motion, which depends on various parameters, such as vessel's size and type, the weather conditions, the location of the LARS on vessel's deck, the size of the LARS, etc. Although it is not possible to measure all the variables, as explained in the next section, the ROV pilot is able to perform the docking maneuver successfully based solely on the visual estimation and prediction of TMS heave displacement. In practice, during manual docking, the ROV pilot estimates the displacement by observing the video feed either from the ROV or the TMS camera to estimate relative motion between the two. A similar approach is presented in this paper, with an ANFIS-based TMS heave displacement prediction $Z_{T M S}$ up to $t$ seconds in the future, based on previous $Z_{T M S}$ measurements. There are various ways to measure TMS heave displacement, such as using depth sensor, altimeter, acoustic positioning system, vision system, etc. As the dataset acquired during the trials consists of TMS depth measurements, and the ROV uses depth control, ANFIS is trained to predict TMS depth. In the next section a manual ROV docking is presented, and importance of TMS heave prediction for the docking is discussed.

\subsection{ROV Docking}

Docking of a ROV system is one of the most critical tasks dictated by operation weather windows. It introduces a high risk of ROV damage, and it can be a highly stressful operation for ROV pilot in challenging sea conditions. The docking maneuver can be divided into three stages: (1) the preparation stage, (2) the ROV approach and TMS heave estimation stage, and (3) contact stage. Manual ROV docking into a cage type TMS starts with the ROV stern facing the entrance of the TMS.

In the first stage, the ROV heading, depth and the lateral position should be aligned with the TMS. In general, the TMS mechanically allows for certain vertical and horizontal misalignment due to the funnel-shaped entrance. Therefore, for relatively small TMS heave amplitudes, approximately heave $_{\max } \leq 1 \mathrm{~m}$ peak-to-peak for the system presented in the paper, the ROV is able to dock while holding mean TMS depth. Figure 3 shows the TMS heaving prior to a manual docking maneuver, whereas the ROV holds constant depth.

However, heave $\max$ is often exceeded, thus in the second stage after the alignment, the vehicle approaches the TMS entrance slowly, while the ROV pilot estimates TMS heave amplitude and frequency. Generally, the work-class ROVs are not agile enough to match the TMS heave motion due to the weight, and high drag forces associated with the ROV's large cross-sectional area. To overcome the problem, the pilot positions the ROV to the docking depth that covers either top or the bottom half of the TMS heave range, as shown in Figure 4. As the TMS reaches the minimum or the maximum heave 
value, it slows down, until it entirely stops and reverses direction. The pilot exploits this knowledge and positions the ROV at a corresponding depth, as the shaded area in Figure 4 shows.
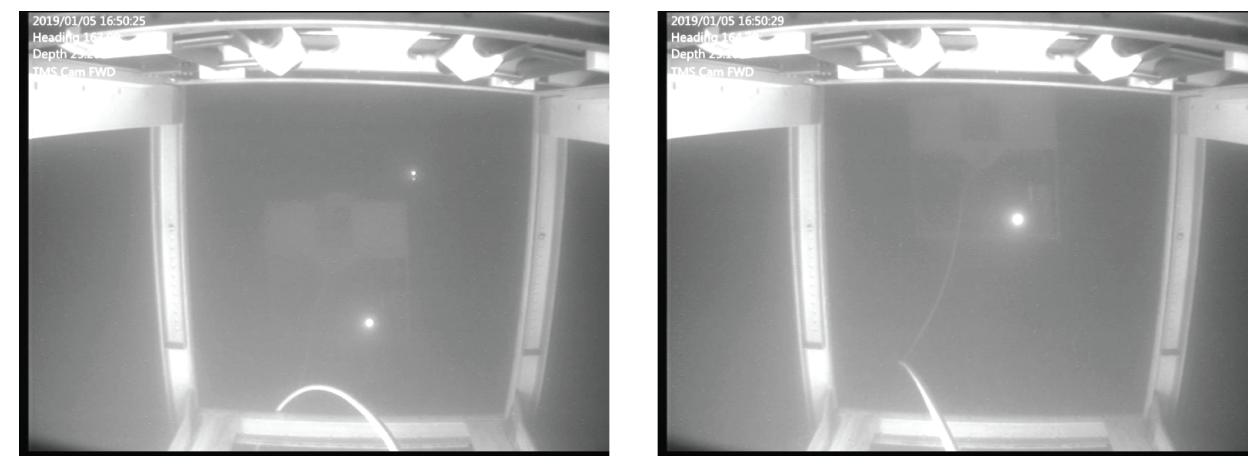

Figure 3. The TMS heaving while ROV holds constant depth. Photo taken during the trials.

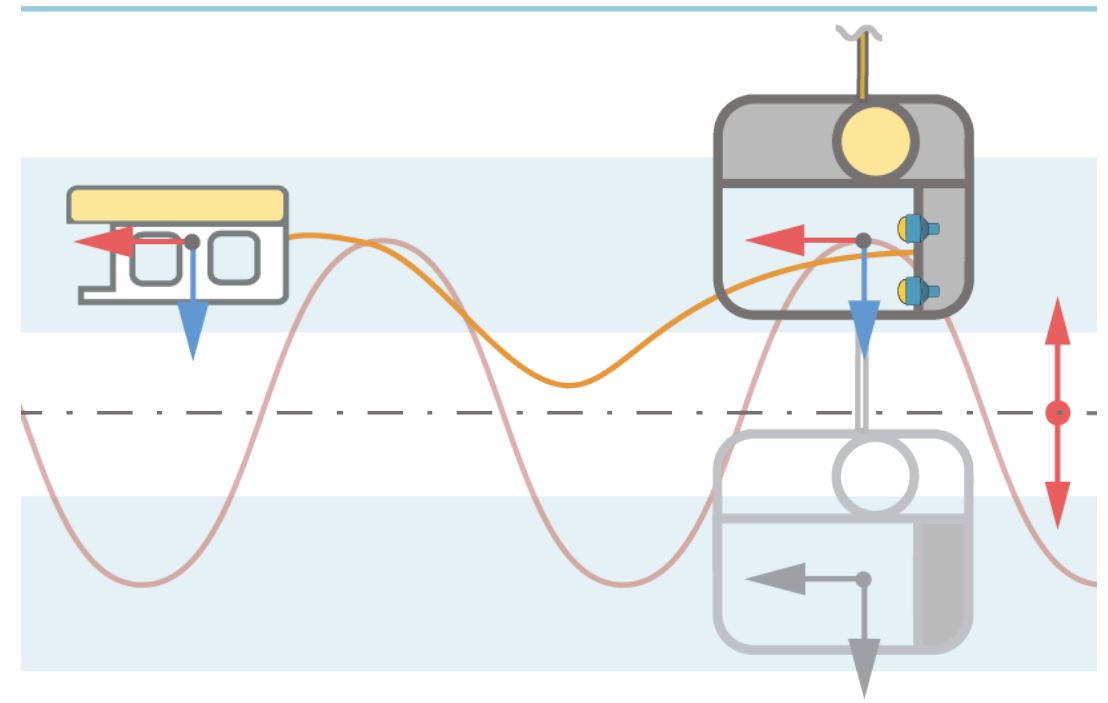

Figure 4. The ROV docking procedure. Red line presents the TMS heave motion, whereas blue shaded area shows optimal docking position with minimal TMS heave speed.

Although the TMS heave amplitude and frequency are not fixed, once the ROV is in the approximately correct area, the ROV depth can be fine adjusted quickly. To allow for large ROV inertia, the docking maneuver is typically started before the TMS reaches the optimal position for docking. Therefore, the pilot must predict the TMS position based on experience, and current and previous observations, and undertake a decision in a fraction of second while controlling the ROV.

The third stage includes the contact between the ROV and the TMS, and finishes with the ROV docked. As, in general, it is not possible to compensate for all the motion and align ROV perfectly with the TMS, the docking still includes rough or bumpy contact; however, to a much reduced extent.

\section{Adaptive Neuro-Fuzzy Inference System - ANFIS}

This section describes the implementation of the adaptive neuro-fuzzy inference system (ANFIS) for TMS motion prediction. ANFIS is an adaptive neural network which is equivalent to a fuzzy inference system (FIS) first time introduced in [24]. With ANFIS, a set of fuzzy if-then rules is identified, with membership function parameters tuned through a hybrid learning algorithm.

Figure 5 shows the ANFIS network architecture that consists of five layers. Each of $m$ inputs $(X)$ is assigned with $n$ fuzzy membership functions described with linguistic labels (A), constituting $r$ rules (R). Each node in the first layer is adaptive and specifies the degree to which a given input satisfies the fuzzy membership function related with that node. The first layer is called the "fuzzification" layer, 
while parameters in this layer are called premise parameters. In the second layer a firing strength for each rule is determined. Every node in this layer is fixed and labeled $\pi$, while the node performs multiplication of the incoming signals. Every node in the third layer is fixed and normalizes the firing strengths of the previous layer. The fourth layer is called the "defuzzification" layer. This layer consists of adaptive nodes and it involves computing the weighted consequent value for each given rule. Parameters in this layer are called consequent parameters. The node in the last layer performs summation of all incoming signals.

Various authors reported use of ANFIS for modeling nonlinear functions such as motion prediction of moving targets [25-27], predicting stock market return [28], electricity price forecasting [29], and various other. In addition, ANFIS performs exceptionally well when predicting chaotic time series. This is demonstrated in [24], where comparison between ANFIS, cascaded-correlation neural network, backpropagation MLP, autoregressive model, and other networks have been given. As the position prediction of the TMS belongs to the same class of problems, the use of ANFIS should be considered and evaluated.

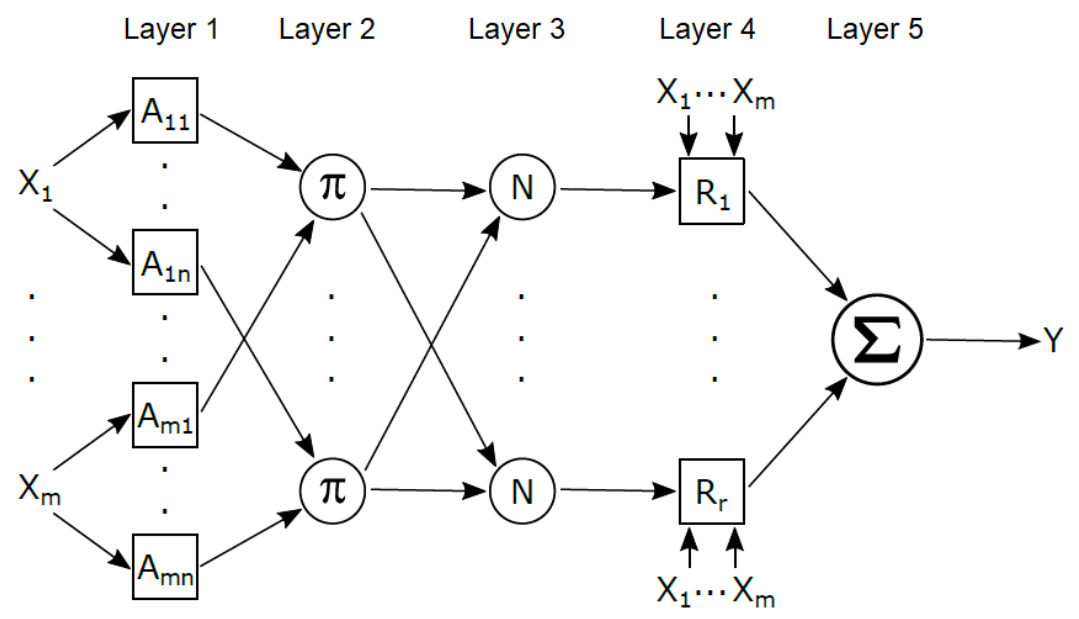

Figure 5. ANFIS network framework architecture.

The tuning of the network is done using an existing dataset consisting of input-output pairs, while the network tries to model the function which relates input to output. By using past values of the heave displacement $z_{T M S}$ up to time $t$, ANFIS is used to predict the future value of the $z_{T M S}(t+P)$. As $z_{T M S}$ is measured using a depth sensor, this is achieved by mapping a dataset of known TMS depth values using $D$ points of the time series spaced $\Delta$ apart as

$$
\left[z_{T M S}(t-(D-1) \Delta), \ldots, z_{T M S}(t-\Delta), z_{T M S}(t)\right],
$$

to a predicted value in future $z_{T M S}(t+P)$. Therefore, for parameters $D=3, \Delta=1.5, P=2$, one input-output ANFIS pair is given by

$$
\left[z_{T M S}(t-3), z_{T M S}(t-1.5), z_{T M S}(t)\right],\left[z_{T M S}(t+2)\right]
$$

where $\left[z_{T M S}(t-3), z_{T M S}(t-1.5), z_{T M S}(t)\right]$ is the input which consists of the last $D=3$ depth measurements, spaced $\Delta=1.5 \mathrm{~s}$ apart, mapped to the output $\left[z_{T M S}(t+2)\right]$, which presents the predicted TMS depth value $P=2 \mathrm{~s}$ in the future.

The ANFIS training and evaluation has been done on a prerecorded dataset. The data used for ANFIS training and evaluation was recorded during the offshore trials that took place in the North Atlantic Ocean during January 2019. The TMS depth was recorded using a depth sensor attached to the TMS frame. The sensor used during the trials was UV-SVP by Valeport. It is a conventional commercial unit that offers pressure, sound velocity and temperature measurements in one housing. Technical specification of the unit is given in Table 2. 
Table 2. The Valeport UV-SVP sensor technical specifications.

\begin{tabular}{lccc}
\hline & Pressure (bar) & Temperature $\left({ }^{\circ} \mathbf{C}\right)$ & Sound Velocity $(\mathrm{m} / \mathbf{s})$ \\
\hline Operating range & 300 & -5 to +35 & 1375 to 1900 \\
System resolution & $0.001 \%$ of range & 0.001 & 0.001 \\
System accuracy & $\pm 0.01 \%$ of range & \pm 0.01 & \pm 0.02 \\
\hline
\end{tabular}

For the given task, different process values are involved in constructing an efficient and reliable ANFIS network. This variables include size of the input-output dataset pairs (training dataset length), number of membership functions per input $M F$, the number of training epochs $N E$, number of training points $D$, how far in future TMS position is to be predicted $P$, spacing between the points $\Delta$, sensor sampling frequency $f_{s}$, etc.

Although there are guidelines about the ANFIS training process [24,30], as with other neural networks, there are still no specific rules to estimate the optimal parameters for the network training. The parameters can vary greatly and depend on the quality of data and complexity of the problem, thus it relies on trial and error experiments. If such an approach is not possible, various techniques for estimating optimal ANFIS tuning parameters have been presented before [31,32]. Although extensive trial and error experiments have been performed to investigate the effect of various network parameters, the focus of the paper is as follows.

- Evaluate ANFIS performance for TMS position prediction.

- Analyze the network training time, and consider real-time ANFIS training.

- Investigate the influence of the depth sensor sample rate on ANFIS performance.

Therefore, in the next section, an overview of the best network configuration is given at the start, followed by an ANFIS overall performance evaluation.

\section{Results}

The scope of the paper is to investigate and evaluate the usage of an ANFIS network for the prediction of TMS heave motion. Prior to the evaluation, an optimal network configuration and training parameters should be determined. The optimal ANFIS for the given problem achieves minimum error RMSE with a minimum network training duration. The RMSE is a root mean square error between predicted future TMS depth value and measured value at that time, and it is considered as one of network performance measures. As explained in Section 2, the TMS heave motion depends on the LARS type and the deployment vessel type. Once the network is trained, the performance is reduced if certain LARS, TMS, and deployment vessel combination are changed. As the goal is to enable the possibility to retrofit the solution to the existing ROV fleet, network training on-site is necessary. In addition, constantly changing sea conditions should be considered. Although the trained network can perform well for a certain amount of time after training, a change in sea conditions influences the network performance, thus the online ANFIS training is considered and tested.

\subsection{Optimal ANFIS Configuration for TMS Heave Prediction}

To find optimal ANFIS training parameters the experiments included varying the following parameters.

- Dataset length in range 50 to $600 \mathrm{~s}$.

- Number of membership functions MF per input in range 2 to 5.

- Number of previous measurements $D$ in range 1 to 12 .

- Spacing between previous measurements $\Delta$ in range 0.5 to $5 \mathrm{~s}$.

- Number of training epochs NE in range 1 to 250 .

- Prediction time $P$ in range 0.5 to $5 \mathrm{~s}$. 
Although extensive experiments have been conducted to determine the best set of parameters, only main experiments related to the ANFIS parameters estimation have been presented, considering the main scope of the paper.

The effect of each parameter on ANFIS performance and training duration is shown in Table 3. In general, the input selection criteria is based on [33], which is based on the assumption that the ANFIS network with the smallest RMSE after one epoch of training has a better potential to achieve a lower RMSE given more training epochs.

Table 3. Relationship between different parameters, ANFIS training duration, and performance.

\begin{tabular}{cccccc}
\hline Parameter & Training Duration & $R M S E_{C H K}$ & $R M S E_{T R N}$ & Generates Overfitting \\
\hline Dataset length & $\uparrow$ & $\uparrow$ & $\downarrow$ & $\downarrow$ & No \\
Number of MF & $\uparrow$ & $\uparrow$ & $\downarrow$ & $\downarrow$ & Yes \\
Number of D & $\uparrow$ & $\uparrow$ & $\downarrow$ & $\downarrow$ & Yes \\
Prediction time P & $\uparrow$ & - & $\uparrow$ & - & No \\
Number of Epochs & $\uparrow$ & $\uparrow$ & $\downarrow$ & $\downarrow$ & Yes \\
\hline
\end{tabular}

The relationship between the number of training points $D$ and the number of epochs, RMSE, and duration of the training process, is shown in Table 4. The data in the table is divided in two major columns by the number of epochs used for training. The left column provides results after only one epoch of training, whereas the right column shows RMS errors and training duration at the epoch with minimum $R M S E_{C H K}$. As shown in the table, both the training $R M S E_{T R N}$ and the checking $R M S E_{C H K}$ error decreased until $D=4$. For $D=5$, the training error keeps decreasing, while the checking error grows. In addition, the difference between the two grows significantly at $D=5$, which is the sign of network overfitting and must be avoided. Although in general the network performed better after more than 1 epoch, the difference in $R M S E_{C H K}$ is not significant. For example, at $D=4$, after one epoch the checking error is $R M S E_{C H K}=0.0422624 \mathrm{~m}$, and the minimum error is achieved at epoch 63 with $R M S E_{C H K}=0.0421802 \mathrm{~m}$. The difference between the two is negligible, whereas the duration of the training extended ten times from $0.053 \mathrm{~s}$ to $0.53 \mathrm{~s}$. The number of membership functions is $M F=2$ as the increase in $M F$ leads to exponential growth of fuzzy rules, thus the training time grows exponentially. In addition, no reduction in $R M S E_{C H K}$ has been achieved.

Table 4. The relationship between training points $\mathrm{D}$, number of training epochs, RMSE, and duration of the training process.

\begin{tabular}{|c|c|c|c|c|c|c|c|}
\hline \multirow[t]{2}{*}{ D } & \multicolumn{3}{|l|}{ Epoch 1} & \multicolumn{4}{|c|}{ Epoch with Minimal Check Error } \\
\hline & $R M S E_{T R N}(\mathrm{~m})$ & $\operatorname{RMSE}_{\mathrm{CHK}}(\mathrm{m})$ & Duration (s) & Epoch & $R M S E_{T R N}(\mathrm{~m})$ & $R M S E_{T R N}(\mathrm{~m})$ & Duration (s) \\
\hline 2 & 0.103221 & 0.0864964 & 0.03305 & 1 & 0.103221 & 0.0864964 & 0.03305 \\
\hline 3 & 0.0613137 & 0.0577967 & 0.050134 & 189 & 0.0569494 & 0.0560394 & 0.370561 \\
\hline 4 & 0.0374812 & 0.0422624 & 0.052966 & 63 & 0.0337162 & 0.0421802 & 0.526817 \\
\hline 5 & 0.0296526 & 0.0973706 & 0.102934 & 42 & 0.0276815 & 0.0861169 & 1.863842 \\
\hline
\end{tabular}

From extensive experiments, the optimal ANFIS input parameters for the given task are found as $D=4, \Delta=1, N E=1, M F=2$ per input. Therefore, the best network performance is achieved by using the last four consecutive measurements $(D=4)$, spaced one second apart $(\Delta=1)$, using only one training epoch, with two membership functions per input $M F=2$. The same network configuration performed best for various values of prediction time $\mathrm{P}$.

The amount of data used for online training should be taken into consideration as well since a larger training dataset increases the ANFIS training duration. The effect of training dataset length on ANFIS training cycle duration, training $R M S E_{T R N}$, and checking $R M S E_{C H K}$ error is shown in Figure 6. Multiple experiments have been conducted with the training dataset increased from 50 to $600 \mathrm{~s}$. As shown in Figure $6 \mathrm{a}$, for $50 \mathrm{~s}$ of the training data, $R M S E_{T R N}$ is relatively low while $R M S E_{C H K}$ is high, 
which is a sign of network overfitting the training data, and should be avoided. The overfitting is caused by small amount of training data points compared to number of ANFIS modifiable parameters. Up to $200 \mathrm{~s}$ of training dataset, most of the $R M S E_{C H K}$ is reduced. After that point, training duration grows with little improvement in $R M S E_{C H K}$ as shown in Figure $6 \mathrm{~b}$.
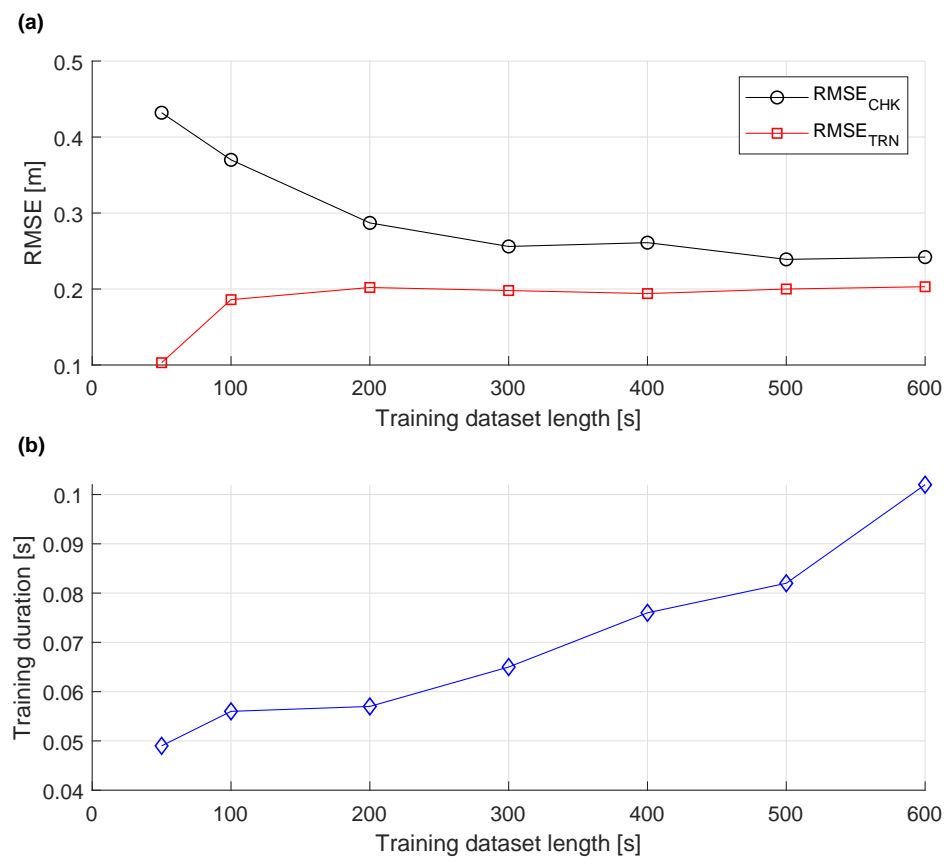

Figure 6. The relationship between length of training dataset and checking error $R M S E_{C H K}$, and training error $R M S E_{T R N}(\mathbf{a})$ and training duration (b).

\subsection{The TMS Heave Prediction Based on ANFIS}

After network parameters ( $D, \triangle, E N, M F$, dataset length) were evaluated as in Section 4.1, multiple tests have been performed to evaluate the ANFIS performance for the TMS heave prediction. Prior to the ANFIS performance test, it is necessary to establish the evaluation criteria. Although hard contact between the ROV and the TMS is expected during the docking in a harsh environment, the main objective is to reduce rough contact to the minimum. This is achieved by reducing the misalignment between the ROV and the TMS at the moment of contact during docking. In general, the TMS and the ROV are designed in such a way to allow a certain amount of misalignment for easier docking. However, this should be minimized to reduce the risk of ROV damage, which leads to increased operational expenditure (OPEX) costs. The amount of allowed misalignment is determined experimentally. During the previously reported offshore trials [22], the work-class ROV was autonomously docked multiple times with the TMS peak to peak heave amplitude of $1.1 \mathrm{~m}$, while the ROV operated at mean TMS depth. Therefore, the particular ROV-TMS configuration shown in Figure 1, and Figure 3 tolerates vertical misalignment of $\pm 0.55 \mathrm{~m}$. Thus, the ANFIS is considered as performing well when the difference between predicted and measured value is:

$$
\operatorname{err}_{T M S}=\left|z_{T M S} p(t+P)-z_{T M S} m(t+P)\right| \leq 0.55 \mathrm{~m},
$$

where $z_{T M S} p(t+P)$ is the predicted TMS depth, and $z_{T M S} m(t+P)$ is the measured TMS depth at the same time. However, sometimes during manual docking the TMS heaves more than the pilot predicts, thus the misalignment between the TMS and the ROV is larger than $\operatorname{err}_{T M S}>0.55 \mathrm{~m}$, and the maneuver has to be aborted. Therefore, as an additional ANFIS performance indicator a mean absolute error (MAE) is calculated as 


$$
M A E=\frac{\sum_{i+1}^{N} a_{i}}{N}
$$

for $a_{i}=1$, if $\operatorname{err}_{T M S_{i}} \leq 0.55 \mathrm{~m}$ and $a_{i}=0$ for $\operatorname{err}_{T M S_{i}}>0.55 \mathrm{~m}$, which essentially shows the percentage of time the error between predicted and measured TMS, depth was $\operatorname{err}_{T M S} \leq 0.55 \mathrm{~m}$. Due to equipment involved in offshore operations being particularly expensive, the network is considered performing well when $M A E \geq 95 \%$.

The depth measurements of the cage type TMS suspended from the ship RV Celtic Explorer during the trials in the North Atlantic Ocean are shown in Figure 7a. The TMS depth recorded over $150 \mathrm{~s}$ period, ranges between 110 and $113 \mathrm{~m}$ with the mean depth of approximately $112 \mathrm{~m}$. The depth sensor sampling frequency was $f_{s}=2 \mathrm{~Hz}$. When working with neural networks, it is common to use a fraction of recorded data for the training, whereas the remaining fraction of the data is used for the network validation. The ANFIS training stage included the first $200 \mathrm{~s}$ of the data, while the next $50 \mathrm{~s}$ of data is used for the checking stage. The experimentally determined optimal ANFIS parameters are $D=4, \Delta=1 \mathrm{~s}, N E=1$, and $M F=2$ per input. Figure $7 \mathrm{~b}$, above, shows only checking data of the same dataset (last $50 \mathrm{~s}$ ) compared to the predicted TMS depth values. The TMS position prediction $1 \mathrm{~s}$ in future $z_{T M S}(t+1)$ (continuous blue line) has the smallest deviation from the measured value, while the difference between checking data and data predicted $3 \mathrm{~s}$ ahead is significant.
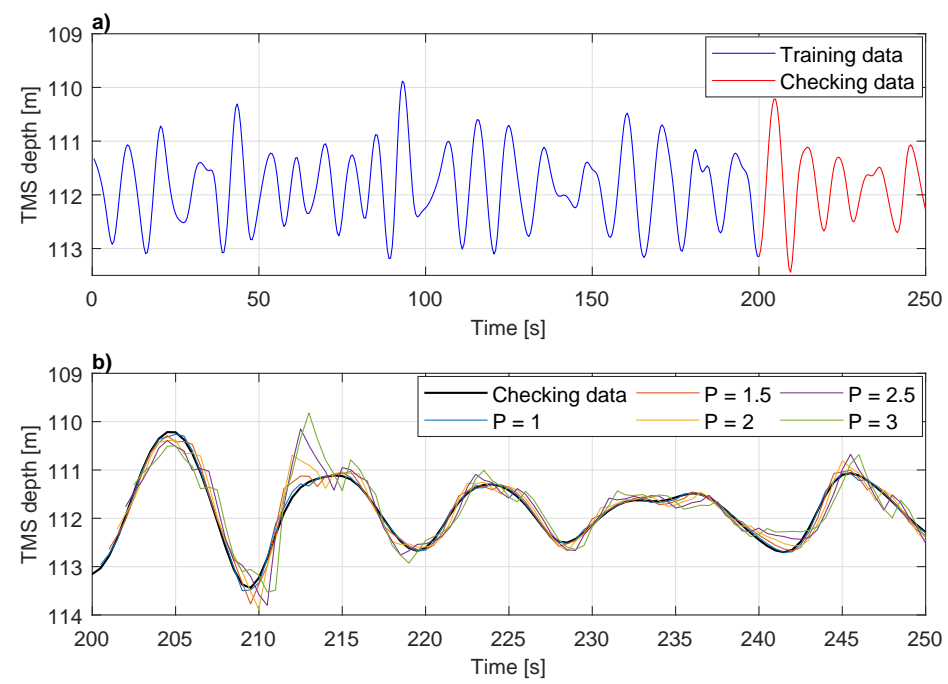

Figure 7. Dataset used for ANFIS evaluation. (a) First $200 \mathrm{~s}$ of data is used for a training, while $50 \mathrm{~s}$ of data is used for checking. (b) Performance of ANFIS predicting TMS depth between $1 \mathrm{~s}$ and $3 \mathrm{~s}$ in future compared to checking data.

Table 5 and Figure 8 show the ANFIS performance for TMS depth prediction up to $P=3 \mathrm{~s}$ in future. ANFIS performs exceptionally well for predicting $z_{T M S}(t+1)$, with two standard deviations of the error only $2 \sigma=0.10 \mathrm{~m}$. For $P=1.5 \mathrm{~s}, 2 \sigma$ reached the value of $0.23 \mathrm{~m}$, and it continues to grow until it reaches the value $2 \sigma=0.55 \mathrm{~m}$ for $P=2.5 \mathrm{~s}$, with $M A E=95.05 \%$. By increasing prediction time further to $\mathrm{P}=3 \mathrm{~s}$, the error grows further, and the criteria $M A E \geq 95 \%$, is not satisfied. Therefore, the results of the experiment showed that ANFIS could be successfully used for the TMS heave position prediction $z_{T M S}$ up to $2.5 \mathrm{~s}$ in future for the particular TMS - ROV setup of the experiments while keeping prediction error below $0.55 \mathrm{~m}$ in $95 \%$ of the time. 

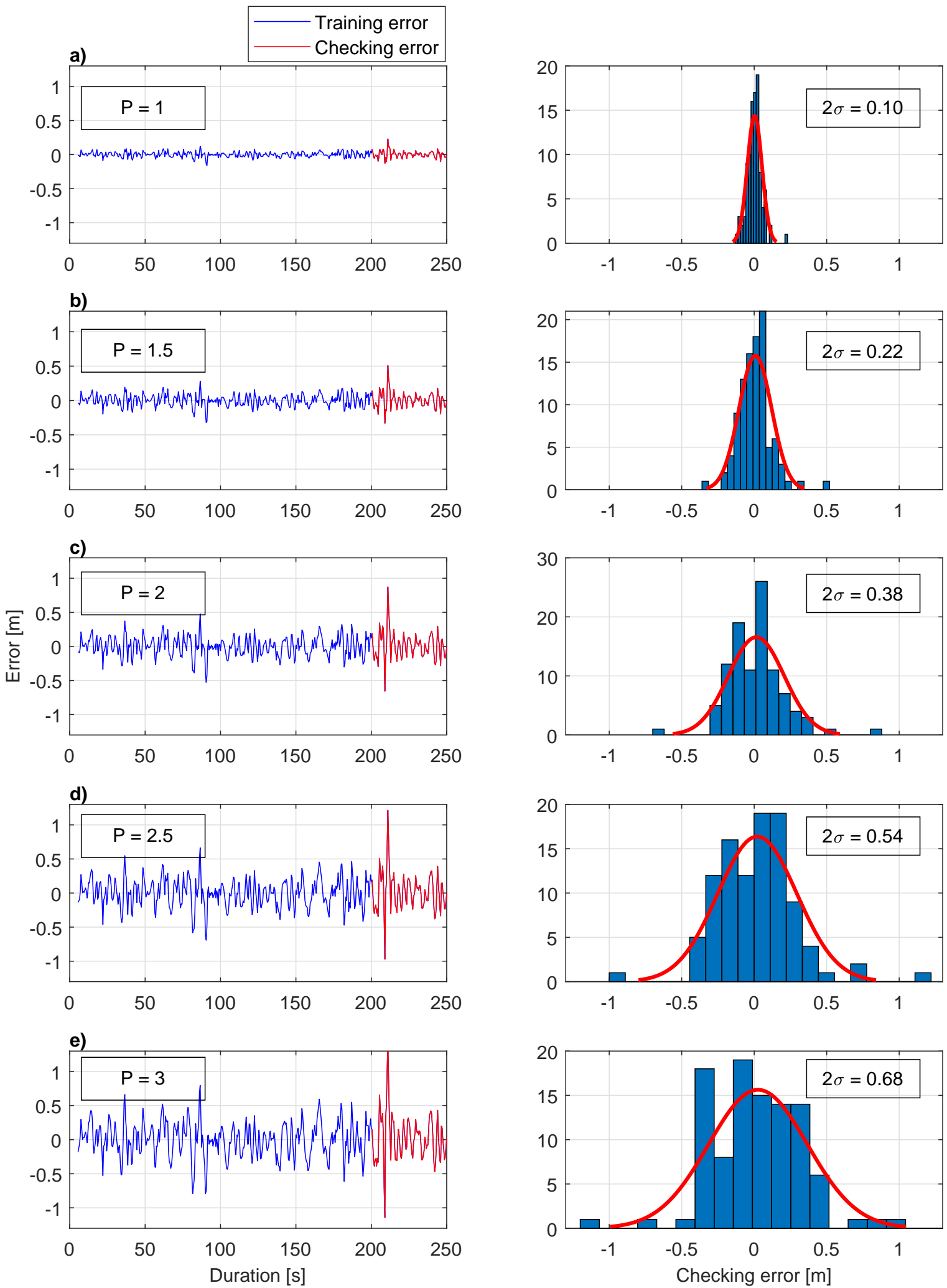

Figure 8. The error between predicted and real TMS depth with corresponding checking error distributions. (a) The TMS depth prediction $1 \mathrm{~s}$ in future; (b) The TMS depth prediction $1.5 \mathrm{~s}$ in future; (c) The TMS depth prediction $2 \mathrm{~s}$ in future; (d) The TMS depth prediction $2.5 \mathrm{~s}$ in future; (e) The TMS depth prediction $3 \mathrm{~s}$ in future. 
Table 5. ANFIS performance for predicting TMS depth up to $P=3 \mathrm{~s}$ in future.

\begin{tabular}{ccccc}
\hline $\mathbf{P}(\mathbf{s})$ & MAE (\%) & RMSE $_{\text {CHK }}(\mathrm{m})$ & $\mathrm{RMSE}_{\text {TRN }}(\mathrm{m})$ & $\mathbf{2 \sigma}(\mathbf{m})$ \\
\hline 1 & 100 & 0.050 & 0.038 & 0.101 \\
1.5 & 99.01 & 0.112 & 0.083 & 0.225 \\
2 & 97.03 & 0.192 & 0.142 & 0.384 \\
2.5 & 95.05 & 0.273 & 0.202 & 0.546 \\
3 & 93.07 & 0.340 & 0.250 & 0.680 \\
\hline
\end{tabular}

\subsection{Online ANFIS Training}

The performance of the ANFIS network is further investigated. As mentioned previously, the sea conditions continuously change, thus the performance of the network trained on one set of the data degrades with changes in TMS heave frequency and/or amplitude. Figure 9a shows the TMS depth recorded over a $900 \mathrm{~s}$ period using the depth sensor sampling frequency $f_{s}=2 \mathrm{~Hz}$. Three ANFIS networks, composing of the same structure $(D=4, M F=2, \Delta=1)$, are trained to predict the TMS depth $2.5 \mathrm{~s}$ in future $(P=2.5)$.

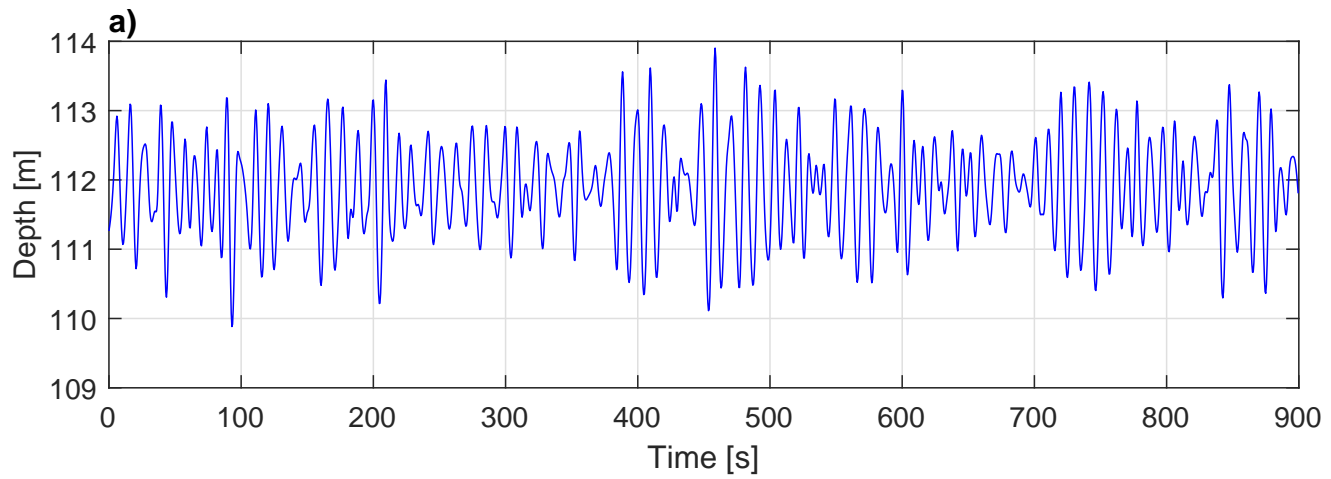

b)

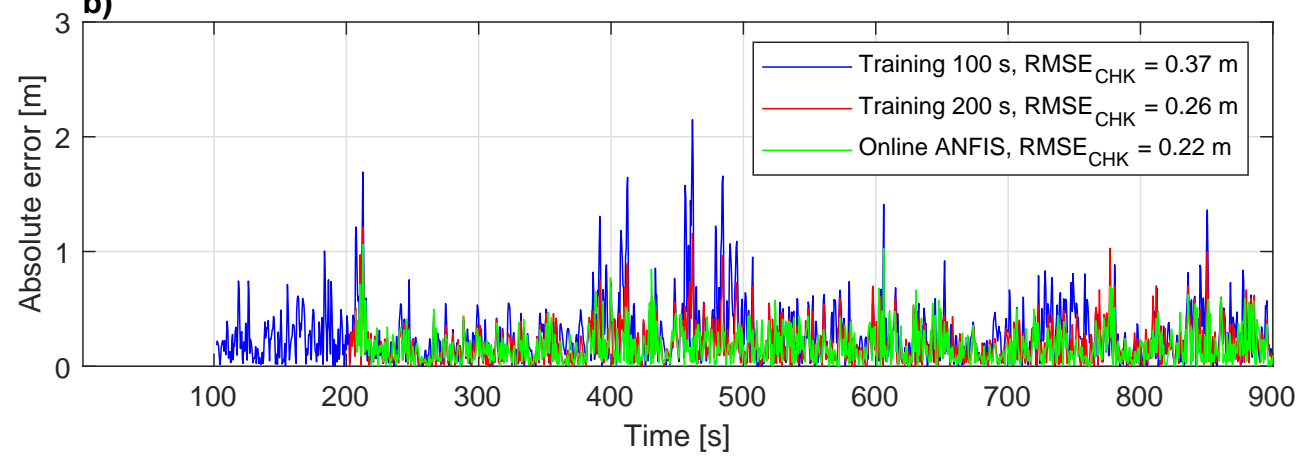

Figure 9. Online trained ANFIS performance. (a) The TMS depth dataset used for ANFIS training and evaluation; (b) Comparison between ANFIS trained on first $100 \mathrm{~s}$ of data (blue), trained on first $200 \mathrm{~s}$ of data (red), and trained online using last $200 \mathrm{~s}$ of data before each prediction step (green).

The first ANFIS network is trained using only the first $100 \mathrm{~s}$ of the data, which means that the prediction model of the TMS behavior has been built based only on those measurements. Similar to other neural networks, once a new input data is out of the range the neural network has been trained for, big errors occur. Therefore, as shown in Figure 9b, at time $200 \mathrm{~s}$, between 380 and $420 \mathrm{~s}$, and between 450 and $500 \mathrm{~s}$ there is a significant error (blue line). For comparison, the second ANFIS network (red line) has been trained using the first $200 \mathrm{~s}$ of the data, therefore it is more "experienced", and has been able to predict the TMS behavior better than the first one. However, a sudden change in TMS depth amplitude between 380 and $420 \mathrm{~s}$, and between 450 and $500 \mathrm{~s}$, still caused high prediction errors. To 
compensate for this, the ANFIS network should be trained considering the latest available data. With the online ANFIS (green line), the prediction model of the TMS behavior has been recalculated and updated after each TMS depth measurement, using the latest $200 \mathrm{~s}$ of the data. For example, at time $380 \mathrm{~s}$ the online ANFIS gives approximately the same error as the second ANFIS, however, at time $420 \mathrm{~s}$ the prediction error has been significantly reduced. Therefore, at time $450 \mathrm{~s}$ based on the last $200 \mathrm{~s}$ of "experience", which also includes the depth measurements between 380 and $420 \mathrm{~s}$, the ANFIS already "expects" a sudden change in the TMS depth, thus between 450 and $500 \mathrm{~s}$ the prediction error is reduced. In summary, with the online ANFIS training approach the network is trained continuously, while taking into account the latest acquired data from the TMS depth sensor. As the figure shows, the online ANFIS training further improved TMS depth prediction. While overall $R M S E_{C H K}$ is reduced (only $0.04 \mathrm{~m}$ ), the error spikes are significantly reduced.

\subsection{Depth Sensor Sample Rate}

In the previous subsection, the optimum training dataset duration is experimentally identified to be $200 \mathrm{~s}$. However, the amount of data points recorded during the $200 \mathrm{~s}$ time period depends on the depth sensor sampling frequency $f_{s}$. Ideally, the sampling frequency of the sensor should be high enough to accurately capture relevant frequency specter, but not too high to cause long ANFIS training time.

Figure 10 shows a frequency specter of the dataset previously illustrated in Figure 9a. The frequency range of the TMS heave motion $f_{T M S}$ is between 0.05 and $0.25 \mathrm{~Hz}$, with the most prominent frequencies $f_{T M S}$ is tetween 0.08 and $0.1 \mathrm{~Hz}$. Therefore, the minimum sensor sampling frequency to cover full TMS heave frequency specter, is by Shannon-Nyquist theorem $f_{s} \min =f_{T M S} \max * 2=0.5 \mathrm{~Hz}$. This was further inspected. Table 6 . shows the relation between ANFIS performance and different sensor sampling frequencies. In each case, the network was trained with $200 \mathrm{~s}$ of data and evaluated on the remaining fraction using the same parameters as follows, $D=4, \Delta=1, N E=1, M F=2$, and $P=2$.

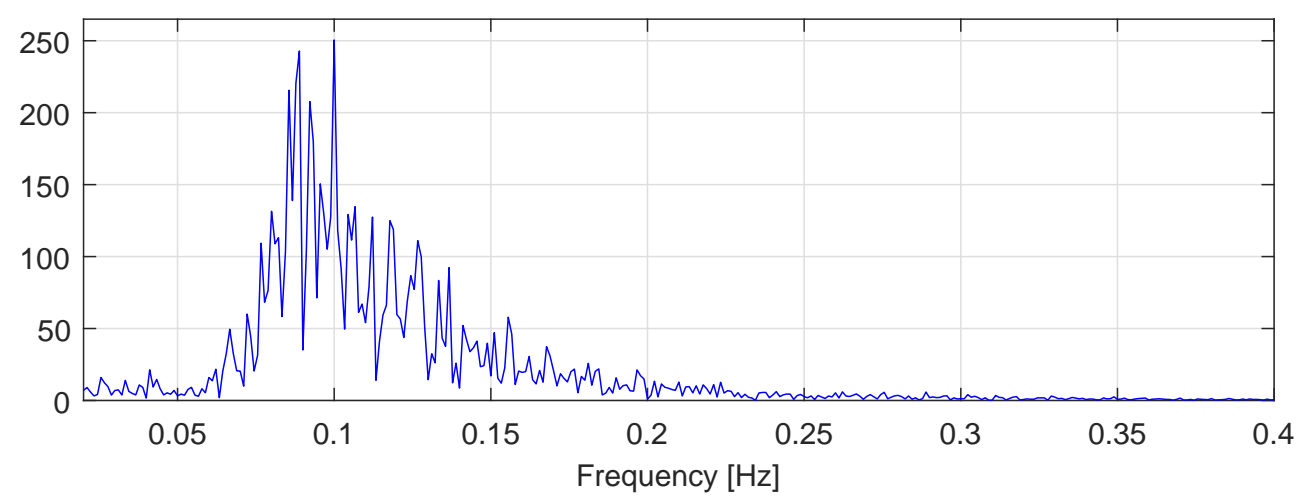

Figure 10. The TMS heave frequency spectre.

Table 6. The relationship between depth sensor sampling frequency and ANFIS performance.

\begin{tabular}{ccccc}
\hline $\begin{array}{c}\text { Sampling Frequency } \\
f_{\boldsymbol{s}}(\mathbf{H z})\end{array}$ & $\begin{array}{c}\text { Number of Datapoints } \\
\text { in 200 s }\end{array}$ & $\begin{array}{c}\text { Training Duration } \\
\mathbf{( s )}\end{array}$ & $\begin{array}{c}\text { RMSE }_{\text {TRN }} \\
(\mathbf{m})\end{array}$ & $\begin{array}{c}\text { RMSE }_{\text {CHK }} \\
(\mathbf{m})\end{array}$ \\
\hline 0.25 & 50 & 0.032 & 0.007 & 2.397 \\
0.5 & 100 & 0.035 & 0.152 & 0.543 \\
1 & 200 & 0.041 & 0.179 & 0.280 \\
2 & 400 & 0.060 & 0.142 & 0.176 \\
4 & 800 & 0.079 & 0.141 & 0.179 \\
8 & 1600 & 0.144 & 0.158 & 0.156 \\
16 & 3200 & 0.322 & 0.158 & 0.157 \\
\hline
\end{tabular}


With the sensor sampling frequency lower than $f_{s}$ min, the actual TMS depth change is not recorded accurately. In addition, the low number of datapoints leads to overfitting, with $R M S E_{C H K}$ over $2 \mathrm{~m}$ at $f_{s}=0.25 \mathrm{~Hz}$. With the increase of the sampling frequency to $f_{s}=f_{s} \min =0.5 \mathrm{~Hz}$, the number of datapoints doubled, and overfitting is avoided. By doubling $f_{s}$ to $1 \mathrm{~Hz}, R M S E_{C H K}$ is further reduced to $0.25 \mathrm{~m}$, which is a big improvement over the previous case. The sensor sampling frequency $f_{s}=2 \mathrm{~Hz}$ provided best results with $R M S E_{C H K}=0.179 \mathrm{~m}$, whereas the ANFIS training time cost is only $0.057 \mathrm{~s}$, and compared to the prediction time of $2.5 \mathrm{~s}$ this is negligible. Further increase in sensor sampling frequency leads to an increase in the ANFIS training duration, while the contribution in prediction performance is minimal.

\section{Discussion and Conclusions}

This paper presents a suspended TMS depth prediction method for ROV docking, based on the Adaptive Neuro-Fuzzy Inference System (ANFIS). The method is used to extend the ROV operational weather windows, reduce operational expenses, and reduce ROV damage due to the harsh docking. The docking of underpowered work-class ROVs to a heaving TMS relies entirely on the ROV pilot experience in estimating TMS heave motion, which is not available for autonomous and resident underwater vehicles. With large ROV inertia and drag forces acting against it, the ROV is not agile enough to match TMS heave motion, thus the docking Figure starts before the ROV and the TMS align. The method presents an addition to the suite of technologies required for dynamic autonomous work-class ROV docking and is beyond the current state of the art in work-class ROV technology. In addition, the method has the potential for retrofitting to the existing ROV fleet, to be used as a ROV pilot aiding tool, and it does not require additional hardware.

The method has been tested in the field on real-world data recorded during the offshore trials in North Atlantic Ocean, during January 2019. The trials during research cruise CE-19001 [34] included work-class ROV Étaín, with the corresponding TMS deployed from the research vessel RV Celtic Explorer, using A-frame LARS. The trained ANFIS network showed excellent performance when predicting the TMS depth up to $2.5 \mathrm{~s}$ into the future with $R M S E=0.22 \mathrm{~m}$, and with $97 \%$ of errors below maximum allowed vertical misalignment between the ROV and the TMS err $\mathrm{TMS}_{i} \leq 0.55 \mathrm{~m}$. Further modification of the TMS entrance with a funnel-shaped receptacle would allow for larger misalignment, thus, extending the operational docking window.

The future work includes detailed time response ROV analysis, modeling the ROV docking strategy decision process, and implementation with the ROV and OceanRings [35,36] suite of smart technologies developed in house at the Centre for Robotics and Intelligent Systems, to extend the ROV autonomous docking capability.

Author Contributions: Conceptualization, P.T. and G.D.; methodology, P.T., G.D., and E.O.; software, P.T.; validation, E.O., G.D., and D.T.; investigation, P.T.; writing-original draft preparation, P.T.; writing-review and editing, P.T., G.D., D.T.; supervision, G.D., E.O., and D.T.; project administration, G.D. and D.T.; funding acquisition, D.T. and G.D. All authors have read and agreed to the published version of the manuscript.

Funding: This material is based upon works supported by Science Foundation Ireland and industry partners Shannon Foynes Port Company and The Commissioners of Irish Lights under the MaREI Research Centres Awards 12/RC/2302_P2 \& 14/SP/2740, RoboVaaS EU ERA-Net Co-fund award through Irish Marine Institute and EU Horizon 2020 research and innovation programme project EUMarineRobots under grant agreement 731103.

Conflicts of Interest: The authors declare no conflicts of interest. The funding sponsors had no role in the design of the study; in the collection, analyses, interpretation of data; or in the writing of the manuscript. 


\section{Abbreviations}

The following abbreviations are used in this manuscript:

ANFIS Adaptive neuro-fuzzy inference system

ASV Autonomous surface vehicle

DOF Degree of freedom

DP Dynamic positioning

FIS Fuzzy inference system

LARS Launch and recovery system

MAE Mean average error

MRE Marine renewable energy

O\&G Oil and gas

OPEX Operating expenses

RMSE Root mean squared error

ROV Remotely operated vehicle

TMS Tether management system

UUV Unmanned underwater vehicle

\section{References}

1. McLeod, D. Emerging capabilities for autonomous inspection repair and maintenance. In Proceedings of the OCEANS 2010 MTS/IEEE SEATTLE, Seattle, WA, USA, 20-23 September 2010.

2. Maslin, E. A Sliding Scale of Residency. Offshore Eng. Mag. 2019, 44, 68.

3. Society for Underwater Technology. Resident E-ROV. UT3 Mag. 2018, 12-4, 30-37.

4. MacDonald, A.; Torkildsen, S.E. ROV in Residence. Offshore Eng. Mag. 2019, 44, 50-53.

5. Trslic, P.; Rossi, M.; Sivcev, S.; Dooly, G.; Coleman, J.; Omerdic, E.; Toal, D. Long term, inspection class ROV deployment approach for remote monitoring and inspection. In Proceedings of the OCEANS 2018 MTS/IEEE Charleston, Charleston, SC, USA, 22-25 October 2018.

6. Albiez, J.; Joyeux, S.; Gaudig, C.; Hilljegerdes, J.; Kroffke, S.; Schoo, C.; Arnold, S.; Mimoso, G.; Alcantara, P.; Saback, R.; et al. FlatFish-a compact subsea-resident inspection AUV. In Proceedings of the OCEANS 2015 - MTS/IEEE Washington, Washington, DC, USA, 19-22 October 2015.

7. Maslin, E. Steps Toward Freedom. Offshore Eng. Mag. 2019, 44, 68.

8. Furuholmen, M.; Hanssen, A.; Carter, R.; Hatlen, K.; Siesjo, J. Resident Autonomous Underwater Vehicle Systems-A Review of Drivers, Applications, and Integration Options for the Subsea Oil and Gas Market. In Proceedings of the Offshore Mediterranean Conference and Exhibition, Ravenna, Italy, 20-22 March 2013.

9. Fahrni, L.; Thies, P.R.; Johanning, L.; Cowles, J. Scope and feasibility of autonomous robotic subsea intervention systems for offshore inspection, maintenance and repair. In Proceedings of the Proceedings of the 3rd International Conference on Renewable Energies Offshore (RENEW 2018), Lisbon, Portugal, 8-10 October 2018.

10. Conte, G.; Scaradozzi, D.; Mannocchi, D.; Raspa, P.; Panebianco, L.; Screpanti, L. Experimental testing of a cooperative ASV-ROV multi-agent system. IFAC-PapersOnLine 2016, 49, 347-354. [CrossRef]

11. Trial proves autonomous ROV deployment capability. Available online: https://www.offshore-mag.com/ business-briefs / equipment-engineering/article/16790676/trial-proves-autonomous-rov-deploymentcapability (accessed on 26 January 2020).

12. Chardard, Y.; Copros, T. Swimmer: Final sea demonstration of this innovative hybrid AUV/ROV system. In Proceedings of the 2002 Interntional Symposium on Underwater Technology (Cat. No.02EX556), Tokyo, Japan, 19 April 2002 .

13. Johansson, B.; Siesjö, J.; Furuholmen, M. Seaeye Sabertooth A Hybrid AUV/ROV offshore system. In Proceedings of the OCEANS 2010 MTS/IEEE SEATTLE, Seattle, WA, USA, 20-23 September 2010.

14. Hobson, B.W.; McEwen, R.S.; Erickson, J.; Hoover, T.; McBride, L.; Shane, F.; Bellingham, J.G. The Development and Ocean Testing of an AUV Docking Station for a 21" AUV. In Proceedings of the OCEANS 2007, Vancouver, BC, Canada, 29 September-4 October 2007. 
15. Allen, B.; Austin, T.; Forrester, N.; Goldsborough, R.; Kukulya, A.; Packard, G.; Purcell, M.; Stokey, R. Autonomous Docking Demonstrations with Enhanced REMUS Technology. In Proceedings of the OCEANS 2006, Boston, MA, USA, 18-21 September 2006.

16. Guidelines for Installing ROV Systems on Vessels or Platforms; International Marine Contractors Association (IMCA): London, UK, May 2013.

17. Liu, S.; Xu, H.; Lin, Y.; Gao, L. Visual Navigation for Recovering an AUV by Another AUV in Shallow Water. Sensors 2019, 19, 1889. [CrossRef] [PubMed]

18. Yazdani, A.; Sammut, K.; Lammas, A.; Clement, B.; Yakimenko, O.A. Cooperative Guidance System for AUV Docking with an Active Suspended Docking Station. In Proceedings of the OCEANS 2019-Marseille, Marseille, France, 17-20 June 2019.

19. Sarda, E.I.; Dhanak, M.R. A USV-Based Automated Launch and Recovery System for AUVs. IEEE J. Ocean. Eng. 2017, 42, 37-55. [CrossRef]

20. Raspante, F. Underwater mobile docking of autonomous underwater vehicles. In Proceedings of the 2012 Oceans, Hampton Roads, VA, USA, 14-19 October 2012; pp. 21-24.

21. Conte, G.; Scaradozzi, D.; Mannocchi, D.; Ciuccoli, N. Field Test of an Integrated ASV/ROV Platform. In Proceedings of the 27th International Ocean and Polar Engineering Conference, San Francisco, CA, USA, 25-30 June 2017.

22. Trslic, P.; Rossi, M.; Robinson, L.; O’Donnel, C.W.; Weir, A.; Coleman, J.; Riordan, J.; Omerdic, E.; Dooly, G.; Toal, D. Vision based autonomous docking for work class ROVs. Ocean Eng. 2020, 196, 106840. [CrossRef]

23. Christ, R.D.; Wernli, R.L. The ROV Manual: A User Guide for Remotely Operated Vehicles, 2nd ed.; Butterworth-Heinemann is an imprint of Elsevier: Amsterdam, The Nertherlands, 2014.

24. Jang, J.S. ANFIS: Adaptive-network-based fuzzy inference system. IEEE Trans. Syst. Man Cybern. 1993, 23, 665-685. [CrossRef]

25. De Costa Sousa, J.; Setnes, M. Fuzzy predictive filters in model predictive control. IEEE Trans. Ind. Electron. 1999, 46, 1225-1232. [CrossRef]

26. Rajpurohit, V.S.; Pai, M.M.M. Efficient Object Motion Prediction Using Adaptive Fuzzy Navigational Environment. In Information Technology and Mobile Communication; Das, V.V., Thomas, G., Lumban Gaol, F., Eds.; Springer: Berlin/Heidelberg, Germany, 2011; pp. 1-5. [CrossRef]

27. Sivcev, S.; Trslic, P.; Adley, D.; Robinson, L.; Dooly, G.; Omerdic, E.; Toal, D. Adaptive Neuro-Fuzzy Network Enhanced Automatic Visual Servoing Algorithm for ROV Manipulators. In Proceedings of the OCEANS 2019 MTS/IEEE SEATTLE, Seattle, WA, USA, 27-31 October 2019.

28. Boyacioglu, M.A.; Avci, D. An Adaptive Network-Based Fuzzy Inference System (ANFIS) for the prediction of stock market return: The case of the Istanbul Stock Exchange. Expert Syst. Appl. 2010, 37, 7908-7912. [CrossRef]

29. Yaser, R.G.; Allah, H.R. Short Term Electricity Price Forecasting by Hybrid Mutual Information ANFIS-PSO Approach. Comput. Intell. Electr. Eng. 2019, 10, 63-72.

30. Karaboga, D.; Kaya, E. Adaptive network based fuzzy inference system (ANFIS) training approaches: A comprehensive survey. Artif. Intell. Rev. 2019, 52, 2263-2293. [CrossRef]

31. Shoorehdeli, M.A.; Teshnehlab, M.; Sedigh, A.K. Novel Hybrid Learning Algorithms for Tuning ANFIS Parameters Using Adaptive Weighted PSO. In Proceedings of the 2007 IEEE International Fuzzy Systems Conference, London, UK, 23-26 July 2007.

32. Buragohain, M.; Mahanta, C. A novel approach for ANFIS modelling based on full factorial design. Appl. Soft Comput. 2008, 8, 609-625. [CrossRef]

33. Jang, J.S. Input selection for ANFIS learning. In Proceedings of the IEEE 5th International Fuzzy Systems, New Orleans, LA, USA, 11 September 1996.

34. Vessel Schedules I Marine Institute. Home Page. Available online: http://www.marine.ie/Home/site-area/ infrastructure-facilities / research-vessels /vessel-schedules?language=ga (accessed on 20 December 2019). 
35. Omerdic, E.; Toal, D. OceanRINGS: System concept and applications. In Proceedings of the 2012 20th Mediterranean Conference on Control \& Automation (MED), Barcelona, Spain, 3-6 July 2012.

36. Omerdic, E.; Toal, D.; Dooly, G. OceanRINGS: Smart Technologies for Subsea Operations. In Advanced in Marine Robotics; Gal, O., Ed.; Lambert Acadmic Publishing: Saarbrucken, Germany; 2013; Chapter 11.

(C) 2020 by the authors. Licensee MDPI, Basel, Switzerland. This article is an open access article distributed under the terms and conditions of the Creative Commons Attribution (CC BY) license (http://creativecommons.org/licenses/by/4.0/). 